\title{
Docking Studies of Rauwolfia Serpentina Alkaloids as Insulin Receptor Activators
}

\author{
Jayasree Ganugapati \\ Associate Professor \\ Department of Biotechnology, \\ Sreenidhi Institute of Science \\ and Technology (SNIST), \\ Yamnampet, Ghatkesar, \\ Hyderabad-501301
}

\author{
Aashish Baldwa \\ Department of Biotechnology, \\ Sreenidhi Institute of Science \\ and Technology (SNIST), \\ Yamnampet, Ghatkesar, \\ Hyderabad-501301
}

\author{
Sarfaraz Lalani \\ Department of Biotechnology \\ Sreenidhi Institute of Science \\ and Technology (SNIST), \\ Yamnampet, Ghatkesar, \\ Hyderabad-501301
}

\begin{abstract}
Rauwolfia serpentina also known as Sarpagandha is an integral part of Ayurvedic medical system for over centuries for the treatment of various ailments. The leaves and roots of Rauwolfia serpentina contain alkaloids which are secondary metabolites. Major alkaloids identified are Reserpine, Rauwolfine, Serpentine, Sarpagine, Ajmaline, Yohimbine and Ajmalicine.

Insulin binds to insulin receptors that are present on different cells of the body. Insulin mediates the absorption of glucose into the cell. Insulin receptor belongs to the class of tyrosine kinase receptor. The three dimensional structure of Insulin Receptor was obtained from PDB database and that of the Alkaloids present in Rauwolfia serpentina were downloaded from CHEMSPIDER database. Docking studies of Insulin Receptor with Alkaloids of Rauwolfia serpetina were performed using Arguslab 4.0.1, Autodock 4.0 and Autodock Vina. The analysis of the results of all three docking softwares suggested that few of the alkaloids present in Rauwolfia serpentina may be potential activators of Insulin Receptor.
\end{abstract}

Keywords: Diabetes, Insulin Receptor, Alkaloids, Rauwolfia serpentina, docking.

\section{INTRODUCTION \\ 1.1 Diabetes}

Diabetes is a metabolic disorder in which blood sugar level rises due to lack of insulin which is a hormone that regulates the level of blood sugar. It is one of the major diseases around the world which can result in diabetic retinopathy, neuropathy and many other conditions that may lead to death.

Type 1 diabetes also called as insulin-dependent or juvenile onset diabetes is caused by an auto-immune reaction where the body's defence system attacks the insulin-producing cells. People with this diabetes produce very little or no insulin. It usually occurs in children or young adults. People suffering form type 1 diabetes need injections of insulin every day in order to control the levels of glucose in their blood.

Type 2 diabetes is also called non-insulin dependent diabetes or adult-onset diabetes, and is the major type in all cases of diabetes. It is characterised by insulin resistance and relative deficiency of Insulin. It is often, associated with obesity, which can lead to insulin resistance and elevated levels of blood glucose.

\subsection{Insulin Receptor}

Insulin receptor belongs to the class of tyrosine kinase receptor. The binding of insulin to its receptor causes conformational changes in the receptor leading to the activation of tyrosine kinase beta subunit. Insulin is responsible for phosphorylation of insulin receptor that leads to glucose uptake by the cells. Insulin is secreted by pancreatic islets in response to increase in blood glucose levels. Most cells of the body have insulin receptors which bind the insulin that is present in the blood circulation. When insulin is attached to insulin receptor of the cell, it initiates a cascade of events that mediates the absorbption of glucose from the blood into the cell. [1].

\subsection{Rauwolfia Serpentina}

Rauwolfia serpentina or Sarpagandha is considered to be an integral part of Ayurvedic and Indigenous medical systems for the treatment of various ailments. Alkaloids which are secondary metabolites are the major constituents present in the leaves and roots of Rauwolfia serpentina [2, 3, and 4]. Major alkaloids identified are Reserpine, Rauwolfine, Serpentine, Sarpagine, Yohimbine, Ajmaline and Ajmalicine. Other plants of this genus are also used medicinally in western medicine, Ayurveda, Unani and Folk medicine [5]. The extracts possess antidiabetic activity $[6,7,8,9]$ and prevent Hypertension [10].

\subsubsection{Alkaloids}

Alkaloids are a group of naturally occurring compounds that contain nitrogen atoms in addition to carbon, hydrogen, oxygen and sulphur. Rarely elements such as chlorine, bromine and phosphorus are also present. Bacteria, Fungi, Plants and Animals produce alkaloids, thus they are a group of natural products. [11]

The hypoglycaemic activity of the Rawolfia alkaloids was studied in anaesthetized cats [12] and alloxan-induced diabetic rats [13].

\section{MATERIALS AND METHODS}

The three dimensional structure of Insulin Receptor of Homo sapiens was obtained from Protein Databank (PDB): PDB ID: 1IR3 [14, 15] and that of alkaloids present in Rauwolfia serpentina were obtained from CHEMSPIDER database [16].

\subsection{Preparation of Ligands}

The CID files of the Ligands were obtained from CHEMSPIDER database. The list of the Ligands (Alkaloids) present in R. serpentina initially used are given in Table 1 . 
Table 1: Alkaloids present in Rauwolfia Serpentina

\begin{tabular}{|c|c|c|}
\hline S.No & ALKALOIDS & CHEMSPIDER ID \\
\hline 1. & Sarpagine & 16736014 \\
\hline 2. & Serpentine & 21560 \\
\hline 3. & Ajmalicine & 390541 \\
\hline 4. & Yohimbine & 8622 \\
\hline 5. & Reserpine & 5566 \\
\hline 6. & Ajmaline & 10145712 \\
\hline
\end{tabular}

The energy minimization of the prepared ligands was carried out with Swiss-PDB Viewer V.4.02 [17].

\subsection{Molinspiration}

Molinspiration, an online tool, used to perform QSAR studies in order to identify potential activators of biological targets. It offers free on-line services for calculation of important molecular properties $(\log \mathrm{P}$, polar surface area, number of hydrogen bond donors and acceptors), as well as prediction of bioactivity score for the most important drug targets.

Molinspiration tool was used to calculate properties of ligands such as $\log \mathrm{P}$, molecular weight, $\mathrm{H}$ bond donors and $\mathrm{H}$ bond Acceptors. [18]

\subsection{Lipinski Rule of Five}

Lipinski's Rule of Five is used to evaluate drug likeness, or determine if a chemical compound with a certain pharmacological or biological activity has properties that would make it a orally active drug in humans. The rule describes molecular properties important for a drug's pharmacokinetics in the human body, including theirabsorption, distribution, metabolism, and excretion ("ADME").

Lipinski's rule considers a compound as a drug if it satisfies the following criteria:

- Not more than 5 hydrogen bond donors (nitrogen or oxygen atoms with one or more hydrogen atoms)

- Not more than 10 hydrogen bond acceptors (nitrogen or oxygen atoms)

- A molecular mass not greater than 500 daltons

- An octanol-water partition coefficient $\log P$ not greater than 5

Lipinski's Rule of Five was applied to select probable ligands [19]. The compound that had more than one violation was eliminated from the present study.

\subsection{Active Site Analysis}

Active site is part of an enzyme where substrates bind and undergo a chemical reaction. The active site of an enzyme is usually found in a cleft or pocket that is lined by amino acid residues that participate in recognition of the substrate. Residues that directly participate in the catalytic reaction mechanism are called active site residues [20].
Active site analysis of the Insulin Receptor was carried out using Swiss PDB Viewer (SPDBV) V.4.02 and from the PDB ligand Explorer [21].

\subsection{Docking Studies}

Molecular docking is a study of how two or more molecular structures fit together. It predicts the structure of the intermolecular complex formed between two or more molecules.

To study the nature of interactions, binding mode and selectivity of Insulin receptor with individual alkaloids, docking was carried out with Arguslab 4.0.1, Autodock 4.0 and Autodock VINA.

\subsubsection{Arguslab 4.0.1}

Arguslab 4.0.1 is Molecular modeling and Drug Docking software. It is very flexible and can reproduce crystallographic binding orientations. Arguslab, which provides a user friendly graphical interface and uses ShapeDock algorithm, was used to carry out docking studies of Insulin receptor with the alkaloids.[22].

\subsubsection{Autodock 4.0}

Autodock 4.0 is used predict the interaction of small molecules with macromolecular targets. Autodock performs docking of the ligand to a set of grids (pre-calculated by Autogrid) describing the active site of target protein $[23,24]$.

The energy grid was built within a cubic box of dimensions $50 \times 50 \times 50 \mathrm{~A}^{\circ}$ with a spacing of $0.375 \mathrm{~A}^{\circ}$. The docking was performed based on Lamarckian Genetic Algorithm [25].

\subsubsection{Autodock Vina}

AutoDock Vina is a new open-source program for drug discovery, molecular docking and virtual screening, offering multi-core capability, high performance and enhanced accuracy and ease of use [26].

AutoDock Vina significantly improves the average accuracy of the binding mode predictions compared to AutoDock 4.

\section{RESULTS AND DISCUSSION} 3.1 Molinspiration Results

The Molinspiration data of the compounds was analyzed using Lipinski's Rule of Five. The compound that had more than one violation was eliminated. Results are indicated in Table 2. Reserpine was seen to violate the Lipinski's Rule of Five and was thus not used for Docking Studies. 
Table 2: Molinspiration Data for Alkaloids of Rauwolfia Serpentina

\begin{tabular}{|c|c|c|c|c|c|c|}
\hline COMPOUND (Alkaloid) & $\begin{array}{c}\text { CHEMSPIDER } \\
\text { ID }\end{array}$ & LOG P & $\begin{array}{c}\text { H BOND } \\
\text { ACCEPTOR }\end{array}$ & $\begin{array}{c}\text { H BOND } \\
\text { DONOR }\end{array}$ & $\begin{array}{c}\text { MOL } \\
\text { WT }\end{array}$ & $\begin{array}{c}\text { No. Of } \\
\text { Violations }\end{array}$ \\
\hline Sarpagine & 16736014 & 1.7 & 3 & 3 & 310.3 & 0 \\
\hline Ajmalicine & 390541 & 2.7 & 4 & 1 & 352.4 & 0 \\
\hline Yohimbine & 8622 & 2.9 & 4 & 2 & 354.4 & 0 \\
\hline Serpentine & 21560 & 3.1 & 3 & 1 & 349.4 & 0 \\
\hline Ajmaline & 10145712 & 1.8 & 4 & 2 & 326.4 & 0 \\
\hline Reserpine & 5566 & 4 & 10 & 1 & 608.6 & 1 \\
\hline
\end{tabular}

\subsection{Active Site Residues}

Active site analysis of the Insulin Receptor was carried out using Swiss PDB Viewer V4.02 and PDB ligand explorer.
The active site consists of seven residues: SER 1006, LYS 1030, GLU 1077, ASP 1083, ASN 1137, ASP 1150, MET 1079 (Figure 1).

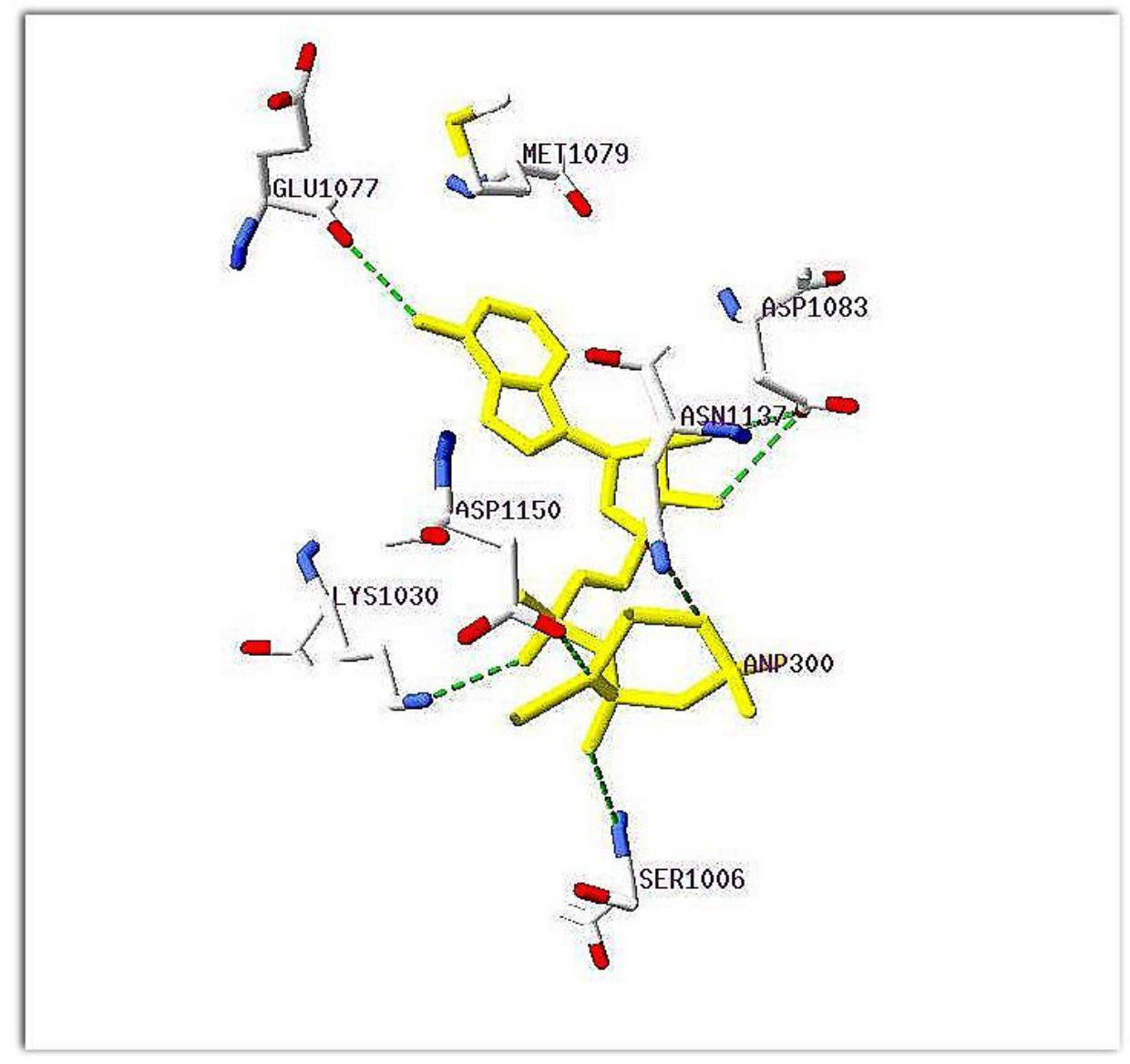

Figure 1: Active site of Insulin Receptor and active site residues are labelled

\subsection{Docking Results:}

The binding mode and interactions (Figure 2 and 3 ) of the selected ligands were analysed according to the binding energies obtained by all the three softwares. The binding energies (Table 3) indicate that Ajmalacine energy value from Arguslab was found to be $-9.89 \mathrm{kcal} / \mathrm{mol}$, that from Autodock 4.0 was found to be $-6.26 \mathrm{kcal} / \mathrm{mol}$ and Autodock Vina it is $8.6 \mathrm{kcal} / \mathrm{mol}$. Ajmalicine was seen to interact with active site residue ASP 1150 by means of hydrogen bond. The energy value of Serpentine from Arguslab was found to be $-9.57 \mathrm{kcal} / \mathrm{mol}$, Autodock 4.0 was found to be $-6.79 \mathrm{kcal} / \mathrm{mol}$ and from Autodock Vina it is $-8.5 \mathrm{kcal} / \mathrm{mol}$. Serpentine was seen to interact with active site residue LYS 1130 by means of hydrogen bond. The energy value of Yohimbine from Arguslab was found to be $-9.29 \mathrm{kcal} / \mathrm{mol}$, that from Autodock 4.0 was found to be $-6.76 \mathrm{kcal} / \mathrm{mol}$ and that from Autodock Vina was found to be $-8.5 \mathrm{kcal} / \mathrm{mol}$. Yohimbine was seen to interact with active site residue SER 1006 by means of 
hydrogen bond. The energy value of Ajmaline from Arguslab was found to be $-9.28 \mathrm{kcal} / \mathrm{mol}$, that from Autodock 4.0 was found to be $-6.15 \mathrm{kcal} / \mathrm{mol}$ and that from Autodock Vina was found to be $-7.9 \mathrm{kcal} / \mathrm{mol}$. Ajmaline was seen to interact with active site residue ASN 1137 by hydrogen bond interactions. The energy value of Sarpagine from Arguslab was found to be $-8.77 \mathrm{kcal} / \mathrm{mol}$, that from Autodock 4.0 was found to be $-7.53 \mathrm{kcal} / \mathrm{mol}$ and that from Autodock Vina was found to be- $8.5 \mathrm{kcal} / \mathrm{mol}$ (Table 3). Sarpagine was seen to interact with active site residues LYS 1030, ASN 1137 and ASP 1150.

Table 3: Energy value (kcal/mol) of docked ligands

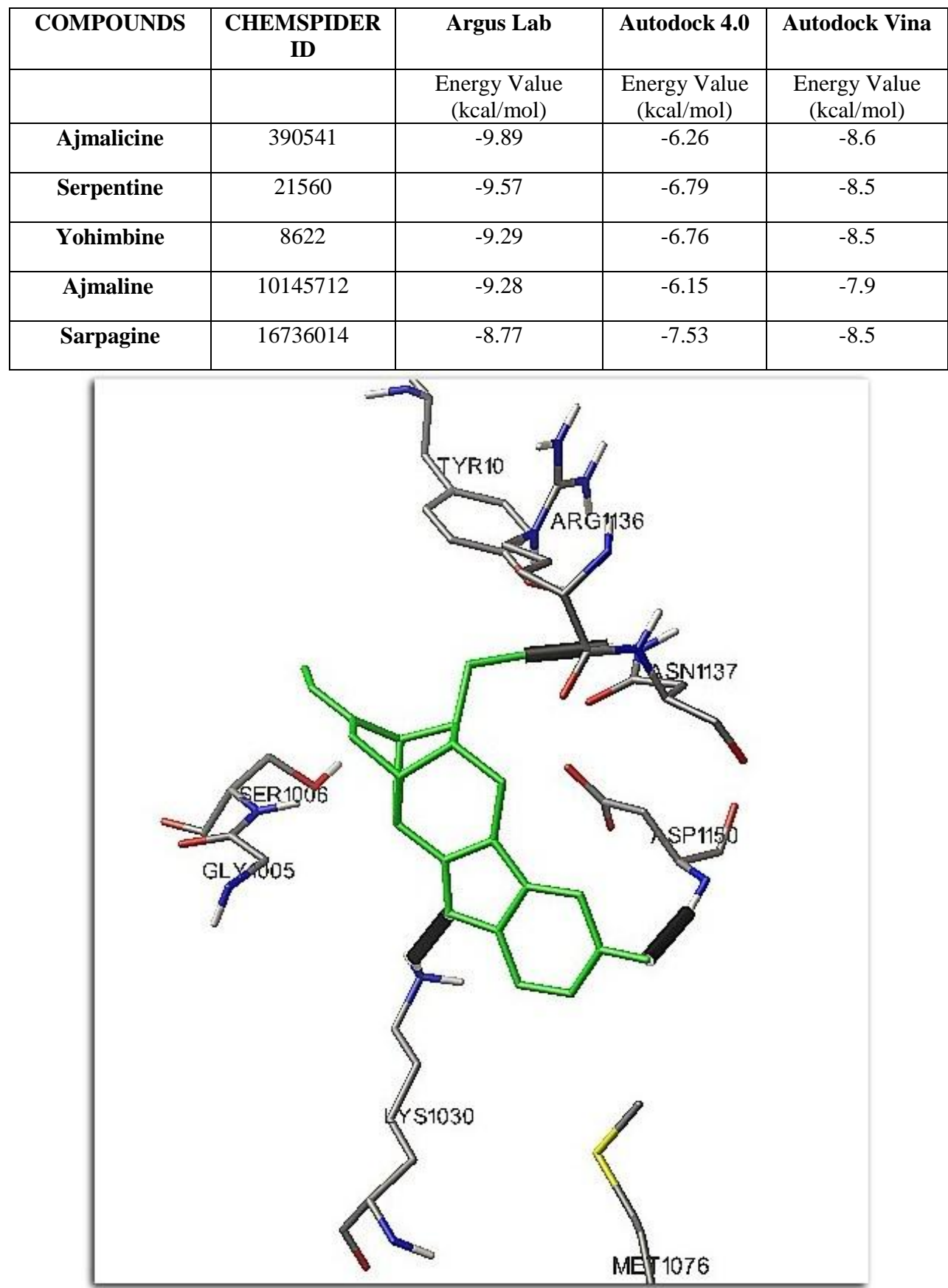

Figure 2: Sarpagine interaction with Insulin Receptor; picture taken by PMV 1.5.4 and docking done by Autodock 4.0. The Compound in green stick model represents the Ligand and rest of the molecule in the stick model are the active site residues (labelled) to which the Ligand is bound. Hydrogen Bonds between Ligand and the Insulin Receptor are represented by black cylindrical structures. 
a)

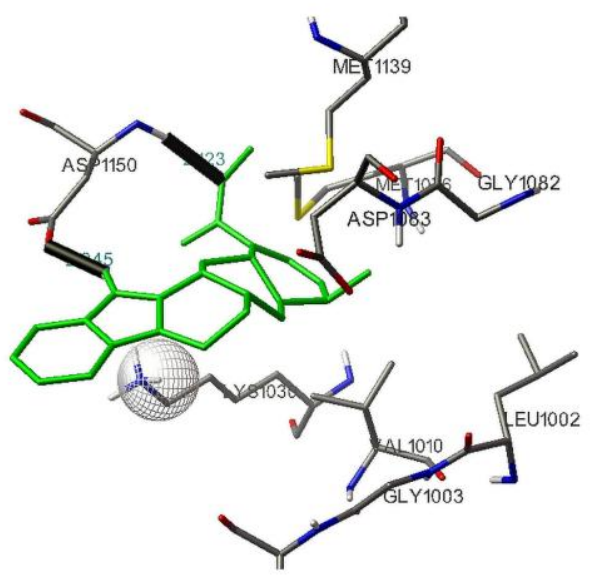

c )

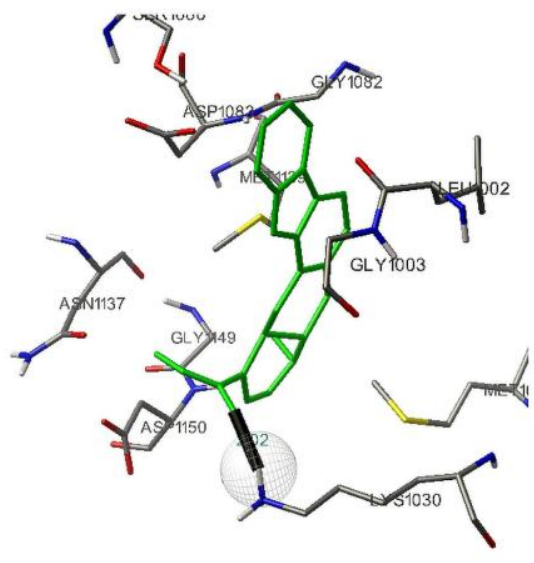

b )

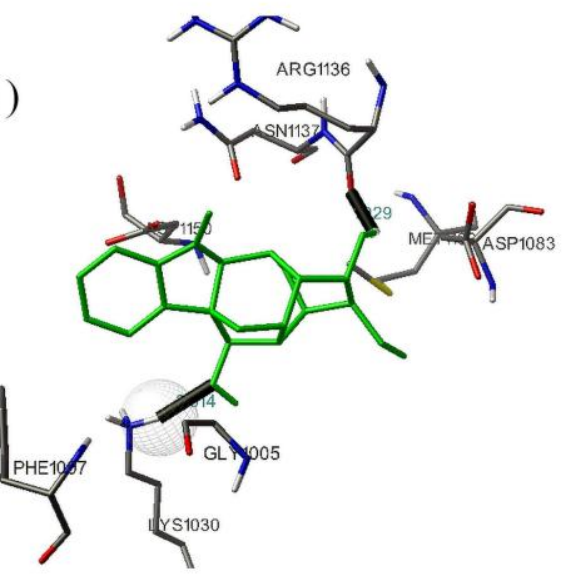

d )

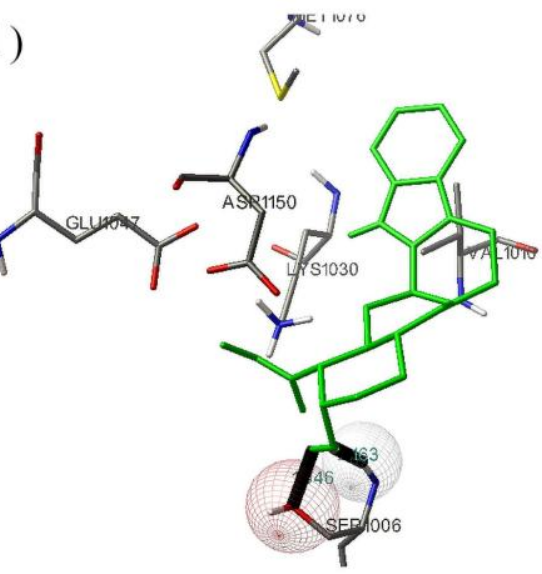

Figure 3: a) Ajmalicine Interaction with Insulin Receptor c) Serpentine Interaction with Insulin Receptor b) Ajmaline Interaction with Insulin Receptor

d) Yohimbine Interaction with Insulin Receptor

\section{CONCLUSION}

The aim of the study was to find out the potent activator of Insulin Receptor. The three dimensional structure of Insulin receptor from PDB database was used for docking studies with R.serpentina alkaloids. Docking results of all three docking software indicates that the selected alkaloids were found to interact with the functional residues of insulin receptor. Hence they can be considered as potent activators. Reserpine had violated one of Lipinski's Rule of Five and was thus not used for docking Studies.

According to the binding energies, all the Alkaloids used for docking possess substantial potential to activate the Insulin Receptor. As these alkaloids are present in various food products and other sources naturally, they can be taken orally either as a supplement or by consuming the food in which they are present and can be used as a remedy to cure diabetes mellitus when there is a deficiency of Insulin, as a substitute or replacement for Insulin. Further analysis can be carried out in the wet lab.

\section{ACKNOWLEDGEMENTS}

We would like to thank the management of Sreenidhi Institute of Science and Technology (SNIST) for their encouragement and support in carrying out the work. Special thanks to Mrs. K. RAJITHA, Department of Biotechnology, Sreenidhi Institute of Science and Technology.

\section{1 and 2 has contributed equally}

\section{REFERENCES}

[1] Ward CW, Lawrence MC (April 2009). "Ligand-induced activation of the insulin receptor: a multi-step process involving structural changes in both the ligand and the receptor". Bioessays 31 (4): 422-34. PMID 19274663

[2] Itoh, A., T. Kumashiro, M. Yamaguchi, N. Nagakura, Y. Mizushina, T. Nishi and T. Tanahashi, 2005. Indole alkaloids and other constituents of Rauwolfia serpentina. J. Nat. Prod., 68: 848-852.

[3] M. W. Klohs, M. D. Draper, F. Keller .Alkaloids of Rauwolfia serpentina Benth. V.1 Rescinnamine.J. Am. Chem. Soc., 1955, 77 (8), pp 2241-: April 1955

[4] R.J. Marles, N.R. Farnsworth Antidiabetic plants and their active constituents. Phytomedicine-Volume 2, Issue 2, October 1995, 137-189

[5] Craig, W.J., 1999. Health promoting properties of common herbs. Am. J. Clin. Nutr., 70: S491-S499.

[6] A.Chauhan, P. K. Sharma, P. Srivastava, N. Kumar, R. Dudhe. Plants Having Potential Antidiabetic Activity: A Review. Der Pharmacia Lettre, 2010, 2(3): 369-387 
[7] Atta-Ur-Rahman, Khurshid Zaman. Medicinal plants with hypoglycemic activity. Journal of Ethnopharmacology-Volume 26, Issue 1, June 1989,155.

[8] Nidhi Aggarwal and Shishu. A Review of Recent Investigations on Medicinal Herbs Possessing AntiDiabetic Properties. J Nutrition Disorder Ther 2011, 1:1.

[9] Satyavati GV, Tandon Neeraj and Sharma Madhu. INDIGENOUS PLANT DRUGS FOR DIABETES MELLITUS

[10] Epstein, M. and J.R. Sowers, 1992. Diabetes mellitus and Hypertension. Hypertension, 19: 403-418.

[11] Dalton, D. R. 2000. Alkaloids. Kirk-Othmer Encyclopedia of Chemical Technology.

[12] Chatterjee, M.L., M.S. De and D. Setb, 1960. Effect of different fractions of Rauwolfia serpentine alkaloids on blood sugar levels in anaesthetized cats. Bull. Call. Sch. Trop. Med., 8: 152-153.

[13] S.A Qureshi, A. Nawaz, S.K. Udani and B. Azmi,, 2009. Hypoglycaemic and hypolipidemic activities of Rauwolfia serpentina in alloxan-induced diabetic rats.. Int. J. Pharmacol., 5: 323-326.

[14] H. M. Berman, J. Westbrook, Z. Feng, G. Gilliland, T. N. Bhat, H. Weissig, I. N. Shindyalov and P. E. Bourne, The Protein Data Bank. Nucleic Acids Res., 28, 235-242, 2000

[15] PDB Database: http://www.rcsb.org/pdb/home/home.do

[16] CHEMSPIDER: http://www.chemspider.com/

[17] GuexN. and Peitsch, M.C. (1997) SWISS-MODEL and the Swiss-PdbViewer: An environment for comparative protein modeling.Electrophoresis 18, 2714-2723.

[18] Molinspiration Cheminformatics, Nova ulica, SK-900 26 Slovensky Grob, Slovak Republic. www.molispiration.com
[19] C. A. Lipinski; F. Lombardo; B. W. Dominy and P. J. Feeney (2001). "Experimental and computational approaches to estimate solubility and permeability in drug discovery and development settings." Adv Drug Del $\operatorname{Rev} 46: 3-26$.

[20] A. D. McNaught and A. Wilkinson. Blackwell Scientific Publications, Oxford (1997).IUPAC: Compendium of Chemical Terminology, 2nd ed. (the "Gold Book"). Compiled by. XML on-line corrected version: (2006-) created by M. Nic, J. Jirat, B. Kosata; updates compiled by A. Jenkins. ISBN 0-9678550-9-8.

[21] Dr. Qing (Cindy) Zhang. Protein Ligand Explorer.

[22] Thompson, M. A. (2004). Molecular docking using ArgusLab, an efficient shape-based search algorithm and the AScore scoring function. ACS meeting, Philadelphia, 172, CINF 42, PA.

[23] Huey, R., Morris, G. M., Olson, A. J. and Goodsell, D. S. (2007), A Semiempirical Free Energy Force Field with Charge-Based Desolvation J. Computational Chemistry, 28: $1145-1152$

[24] Michel F. Sanner. Python: A Programming Language for Software Integration and Development. J. Mol. Graphics Mod., 1999,Vol 17, February. pp57-61

[25] Morris, G. M., Goodsell, D. S., Halliday, R. S., Huey, R., Hart, W. E., Belew, R. K. and Olson, A. J.(1998), Automated docking using a Lamarckian genetic algorithm and an empirical binding free energy function. J. Comput. Chem., 19: 1639-1662

[26] O. Trott, A. J. Olson, AutoDock Vina: Improving the speed and accuracy of docking with a new scoring function, efficient optimization and multithreading, Journal of Computational Chemistry 31 (2010) 455-461 